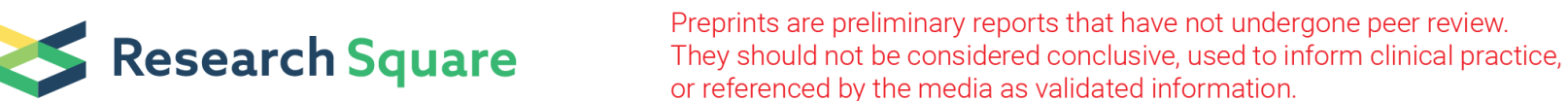

\section{Membrane Protein Metabolism in UNG-Activated NCAPH and RFC4 Subnetworks for Viral HCC|blood Cancer Development Via Inside-Out Microtubule Binding}

\section{Lin Wang ( $\sim$ wanglin98@tsinghua.org.cn )}

Beijing University of Posts and Telecommunications https://orcid.org/0000-0003-2433-8594

\section{Qingchun Chen}

Beijing University of Posts and Telecommunications

\section{Haitao Feng}

Beijing University of Posts and Telecommunications

\section{Minghu Jiang}

Tsinghua University

Juxiang Huang

Beijing University of Posts and Telecommunications

Zhenfu Jiang

Beijing Sougo Technology Development Co. Ltd

\section{Primary research}

Keywords: UNG-activated NCAPH and RFC4 subnetworks, membrane protein metabolism, viral HCC|blood cancer development, inside-out, nucleus microtubule binding

Posted Date: August 28th, 2020

DOI: https://doi.org/10.21203/rs.3.rs-66105/v1

License: (c) (i) This work is licensed under a Creative Commons Attribution 4.0 International License. Read Full License 


\section{Abstract}

Background: Our aim is to compute NCAPH and RFC4 subnetworks containing CDKN3 (feedback) for the novel molecular and cellular mechanisms of HCC (HBV or HCV) related cancer development from high UNG-activated upstream network.

Methods: Non-SMC condensin I complex subunit H (NCAPH) and replication factor $\mathrm{C}$ subunit 4 (RFC4) common molecular and knowledge subnetworks containing cyclin-dependent kinase inhibitor 3-CDKN3 (feedback) related to cancer by references were identified in HCC (HBV or HCV), based on our established significant high expression uracil DNA glycosylase (UNG)-activated upstream Gene (protein) reconstruction network inference (GRNInfer) and Database for Annotation, Visualization and Integrated Discovery (DAVID).

Results: Our results show the common molecules MAPT interaction with CDKN3 with RRM2 with HIST1H3H from UNG-activated upstream GRNInfer database; The common biological process and molecular function of MAPT as microtubule binding; HIST1H3H as cellular protein metabolic process from UNG-activated upstream DAVID database; The common cellular component of UNG, NCAPH, CDKN3, RRM2, HIST1H3H at nucleus; NCAPH, HIST1H3H at membrane; The common tissue distributions of NCAPH and RFC4 in Leukemiapromyelocytic(hl60), leukemialymphoblastic(molt4), etc.

Conclusions: We propose and mutual positively verify membrane protein metabolism in UNG-activated NCAPH and RFC4 subnetworks for viral HCC|blood cancer development via inside-out microtubule binding.

\section{Background}

Cyclin-dependent kinase inhibitor 3 (CDKN3) is not only the more active molecule in our established high uracil DNA glycosylase (UNG)-activated network, but also CDKN3 (feedback) as the common molecule of non-SMC condensin I complex subunit $\mathrm{H}$ (NCAPH) and replication factor $\mathrm{C}$ subunit 4 (RFC4) subnetworks in $\mathrm{HCC}$ (HBV or HCV). CDKN3, NCAPH and RFC4 or the related family molecules have been previously published associations with cancer in the references. Such as, CDKN3 promotes tumor progression through RAD51 in esophageal cancer [1]. CDKN3 regulates cisplatin resistance to colorectal cancer via TIPE1 [2]. Levels of human replication factor $\mathrm{C} 4$ correlate with tumor progression and predict the prognosis for colorectal cancer [3]. DNA replication and sister chromatid cohesion 1 (DSCC1) of the replication factor complex CTF18-RFC is critical for colon cancer cell growth [4]. NCAPH is upregulated in endometrial cancer and associated with poor clinicopathologic characteristics [5]. Overexpression of NCAPH is upregulated and predicts a poor prognosis in prostate cancer [6]. However, NCAPH and RFC4 subnetworks containing CDKN3 (feedback) has not been explored for the novel molecular and cellular mechanisms of HCC (HBV or HCV) related cancer development from high UNG-activated upstream network. 
In the paper, NCAPH and RFC4 feedback/up/downstream molecular subnetworks from our established significant high expression UNG-activated upstream Gene (protein) reconstruction network inference (GRNInfer) [7] database will be constructed in HCC (HBV or HCV) successively by significance analysis of microarrays (SAM) (fold change $\geq 2$ ), Pearson positive correlation coefficient ( $C C \geq 0.25$ ) database with UNG, other mutual positive Pearson correlation (CC $\geq 0.25$ ), respectively. NCAPH and RFC4 common molecular subnetworks containing CDKN3 (feedback) will be computed from high UNG-activated upstream GRNInfer database. NCAPH and RFC4 common biological process, molecular function, cellular component subnetworks containing CDKN3 (feedback) will be computed from high UNG-activated upstream Database for Annotation, Visualization and Integrated Discovery (DAVID) GOTERM_BP_DIRECT, GOTERM_MF_DIRECT, GOTERM_CC_DIRECT $[8,9]$. NCAPH and RFC4 common and different tissue distributions will be calculated from high UNG-activated upstream DAVID GNF_U133A_QUARTILE and UNIGENE_EST_QUARTILE database.

\section{Methods}

225 significant high expression molecules in $25 \mathrm{HCC}$ (HBV or HCV) were identified based on 6144 genes compared with the corresponding low expression of 25 no-tumor hepatitis/cirrhotic tissues (HBV or HCV infection) in GSE10140-10141 [10, 11] (public free from NCBI) by SAM [12] (http://wwwstat.stanford.edu/ tibs/SAM/). Data were processed using a log base of two and two unpaired classes with minimum fold change $(\geq 2)$. A false-discovery rate of $0 \%$ was chosen.

Low and high expression Pearson positive correlation coefficient $(C C \geq 0.25)$ molecules with UNG were calculated in no-tumor hepatitis/cirrhotic tissues (HBV or HCV infection) and HCC (HBV or HCV) from our established Pearson correlation coefficient database of total 225 significant expression molecules by SPSS. Low and high UNG-activated upstream molecular lists in no-tumor hepatitis/cirrhotic tissues (HBV or HCV infection) and HCC (HBV or HCV) were calculated from our established significant low and high expression UNG activation GRNInfer database. GRNInfer is a tool used to construct the activation and inhibition feedback/up/downstream molecular network based on linear programming and decomposition procedure defined by the following equation:

$$
\mathcal{J}=(\dot{\mathrm{X}}-\mathrm{B}) \mathrm{UE}^{-1} \mathrm{~V}^{\mathrm{T}}+\mathrm{YV}^{\mathrm{T}}=\hat{\jmath}+\mathrm{YV}^{\mathrm{T}}
$$

The other mutual positive Pearson correlation $(C C \geq 0.25)$ molecules except UNG were computed in notumor hepatitis/cirrhotic tissues (HBV or HCV infection) based on low UNG-activated upstream molecular list. Low UNG-activated upstream molecular network based on the corresponding mutual positive Pearson correlation database was constructed in no-tumor hepatitis/cirrhotic tissues (HBV or HCV infection) from our established significant low expression UNG activation GRNInfer database.

Low UNG-activated upstream knowledge network was identified in no-tumor hepatitis/cirrhotic tissues (HBV or HCV infection) from our established significant low expression UNG activation DAVID database 
(https://david.ncifcrf.gov/). Low UNG-activated upstream common biological process, molecular function, cellular component network was set up in no-tumor hepatitis/cirrhotic tissues (HBV or HCV infection) from low UNG activation DAVID GOTERM_BP_DIRECT, GOTERM_MF_DIRECT, GOTERM_CC_DIRECT database. Low UNG-activated upstream common tissue distribution network was set up in no-tumor hepatitis/cirrhotic tissues (HBV or HCV infection) from low UNG activation DAVID GNF_U133A_QUARTILE and UNIGENE_EST_QUARTILE database.

The other mutual positive Pearson correlation $(C C \geq 0.25)$ molecules except UNG were computed in HCC (HBV or HCV) based on high UNG-activated upstream molecular list. High UNG-activated upstream molecular network based on the corresponding mutual positive Pearson correlation database was set up in HCC (HBV or HCV) from our established significant high expression UNG activation GRNInfer database. NCAPH and RFC4 feedback/up/downstream direct and indirect molecular subnetwork containing CDKN3 (feedback) in HCC (HBV or HCV) was constructed from our established significant high expression UNGactivated upstream GRNInfer database, respectively. NCAPH and RFC4 common molecular subnetworks containing CDKN3 (feedback) in HCC (HBV or HCV) were computed from our established NCAPH and RFC4 feedback/up/downstream direct and indirect molecular database.

High UNG-activated upstream knowledge network in HCC (HBV or HCV) was identified from our established significant high expression UNG activation DAVID database. NCAPH and RFC4 common biological process, molecular function, cellular component subnetwork containing CDKN3 (feedback) was set up in HCC (HBV or HCV) from our established significant high expression UNG-activated upstream DAVID GOTERM_BP_DIRECT, GOTERM_MF_DIRECT, GOTERM_CC_DIRECT database, respectively. NCAPH and RFC4 common and different tissue distributions were set up in HCC (HBV or HCV) from high UNG-activated upstream DAVID GNF_U133A_QUARTILE and UNIGENE_EST_QUARTILE database.

\section{Results}

NCAPH and RFC4 common molecular subnetworks containing CDKN3 (feedback) from our established significant high expression UNG-activated upstream GRNInfer database were identified as BUB1B (upstream), MAPT (upstream), CDKN3 (feedback), RRM2 (downstream), HIST1H3H (downstream) in HCC (HBV or HCV) successively by SAM and Pearson using GSE10140-10141. CDKN3 activates to CDKN3, CDKN3 to RRM2, CDKN3 to HIST1H3H, CDKN3 to MAPT, MAPT to CDKN3, MAPT to HIST1H3H, MAPT to MAPT, BUB1B to HIST1H3H, HIST1H3H to CDKN3, HIST1H3H to RRM2, RRM2 to CDKN3, RRM2 to RRM2, RRM2 to HIST1H3H, RRM2 to MAPT, RRM2 to BUB1B, as shown in Fig. 1-2.

NCAPH and RFC4 common biological process and molecular function subnetworks containing CDKN3 (feedback) were identified MAPT as microtubule binding; HIST1H3H as cellular protein metabolic process from high UNG-activated upstream DAVID GOTERM_BP_DIRECT and GOTERM_MF_DIRECT database. $\mathrm{NCAPH}$ and RFC4 common cellular component subnetwork containing CDKN3 (feedback) was selected 
UNG, NCAPH, CDKN3, RRM2, HIST1H3H at nucleus; NCAPH, HIST1H3H at membrane in HCC (HBV or HCV) from high UNG-activated upstream DAVID GOTERM_CC_DIRECT database, as shown in Table 1. 
Table 1

NCAPH and RFC4 common biological process, molecular function, cellular component subnetworks containing CDKN3 (feedback) in HCC (HBV or HCV) from our established significant high expression UNG-activated upstream DAVID GOTERM_BP_DIRECT, GOTERM_MF_DIRECT, GOTERM_CC_DIRECT database.

NCAPH and RFC4 common biological process, molecular function, cellular component subnetworks in $\mathrm{HCC}(\mathrm{HBV}$ or $\mathrm{HCV})$

NCAPH subnetwork

UNG (first-core), NCAPH (secondcore), CCNA2 (upstream), ECT2 (upstream), CDKN3 (feedback), KIAA0101 (feedback), NUSAP1 (downstream), RRM2 (downstream), HIST1H3H (downstream), TOP2A (downstream)

BUB1B (upstream), CCNA2 (upstream), ECT2 (upstream), CDKN3 (feedback), TROAP (feedback), GPSM2 (feedback), UBE2C (feedback), KIAA0101 (feedback), NUSAP1 (downstream), RRM2 (downstream)

UNG (first-core), CCNA2 (upstream), RFC4 (upstream), UBE2C (feedback), KIAA0101 (feedback), RRM2 (downstream), HIST1H3H (downstream), TOP2A (downstream)

NCAPH (second-core), BUB1B (upstream), ECT2 (upstream), MAPT (upstream), UBE2C (feedback), RRM2 (downstream)

BUB1B (upstream), CDKN3 (feedback), KIAA0101 (feedback)

NCAPH (second-core), HIST1H3H (downstream)

BUB1B (upstream), UBE2C (feedback)

HIST1H3H (downstream), TOP2A (downstream)

HIST1H3H (downstream), TOP2A (downstream)
RFC4 subnetwork

Terms

UNG (first-core), FOXM1

(upstream), KIAA0101 (upstream),

TOP2A (upstream), NUSAP1

(feedback), CDKN3 (feedback),

ECT2 (feedback), RRM2

(downstream), HIST1H3H

(downstream), NCAPH

(downstream)

BUB1B (upstream), FOXM1

(upstream), GPSM2 (upstream),

cytoplasm

KIAA0101 (upstream), UBE2C

(upstream), NUSAP1 (feedback),

CDKN3 (feedback), ECT2

(feedback), RRM2 (downstream)

UNG (first-core), RFC4 (secondcore), FOXM1 (upstream),

nucleoplasm

KIAA0101 (upstream), TOP2A

(upstream), UBE2C (upstream),

RRM2 (downstream), HIST1H3H

(downstream)

BUB1B (upstream), MAPT

(upstream), UBE2C (upstream),

cytosol

ECT2 (feedback), RRM2

(downstream), NCAPH

(downstream)

BUB1B (upstream), KIAA0101

(upstream), CDKN3 (feedback)

perinuclear region of cytoplasm

HIST1H3H (downstream), NCAPH membrane (downstream)

BUB1B (upstream), UBE2C (upstream)

anaphase promoting complex

TOP2A (upstream), HIST1H3H (downstream)

TOP2A (upstream), HIST1H3H (downstream) nuclear chromosome

protein complex 
NCAPH and RFC4 common biological process, molecular function, cellular component subnetworks in $\mathrm{HCC}(\mathrm{HBV}$ or $\mathrm{HCV})$ core), BUB1B (upstream), CCNA2 (upstream), RFC4 (upstream), CDKN3 (feedback), TROAP (feedback), GPSM2 (feedback), UBE2C (feedback), KIAA0101 (feedback), NUSAP1 (downstream), RRM2 (downstream), HIST1H3H (downstream), TOP2A (downstream)
UNG (first-core), NCAPH (second(upstream), ECT2 (upstream), MAPT

UNG (first-core), RFC4 (second-

NCAPH (second-core), BUB1B

(upstream), CCNA2 (upstream), RFC4 (upstream), UBE2C (feedback), RRM2 (downstream), TOP2A (downstream)

NCAPH (second-core), BUB1B (upstream), CCNA2 (upstream), UBE2C (feedback)

BUB1B (upstream), RFC4 (upstream), UBE2C (feedback), TOP2A (downstream)

BUB1B (upstream), ECT2 (upstream), HIST1H3H (downstream)

RFC4 (upstream), KIAA0101 (feedback), RRM2 (downstream)

NUSAP1 (downstream), HIST1H3H (downstream), TOP2A (downstream)

BUB1B (upstream), MAPT (upstream)

BUB1B (upstream), UBE2C (feedback)

BUB1B (upstream), UBE2C (feedback)

BUB1B (upstream), UBE2C (feedback) core), BUB1B (upstream), FOXM1

(upstream), GPSM2 (upstream),

KIAA0101 (upstream), MAPT

(upstream), TOP2A (upstream),

UBE2C (upstream), NUSAP1

(feedback), CDKN3 (feedback),

ECT2 (feedback), RRM2

(downstream), HIST1H3H

(downstream), NCAPH

(downstream)

RFC4 (second-core), BUB1B (upstream), FOXM1 (upstream), TOP2A (upstream), UBE2C

(upstream), RRM2 (downstream), NCAPH (downstream)

BUB1B (upstream), UBE2C (upstream), NCAPH (downstream)

cell division

RFC4 (second-core), BUB1B

(upstream), TOP2A (upstream), UBE2C (upstream)

BUB1B (upstream), ECT2

(feedback), HIST1H3H (downstream)

mitotic cell cycle

protein binding

RFC4 (second-core), KIAA0101

(upstream), RRM2 (downstream)

FOXM1 (upstream), TOP2A

(upstream), NUSAP1 (feedback), HIST1H3H (downstream)

BUB1B (upstream), MAPT (upstream)

BUB1B (upstream), UBE2C (upstream)

mitotic spindle assembly checkpoint

BUB1B (upstream), UBE2C (upstream)

anaphase promoting complex dependent proteasomal ubiquitin dependent protein catabolic process

BUB1B (upstream), UBE2C (upstream) negative regulation of ubiquitin protein ligase activity involved in mitotic cell cycle 
NCAPH and RFC4 common biological process, molecular function, cellular component subnetworks in $\mathrm{HCC}(\mathrm{HBV}$ or $\mathrm{HCV})$

$\begin{array}{lll}\text { BUB1B (upstream), UBE2C (feedback) } & \begin{array}{l}\text { BUB1B (upstream), UBE2C } \\ \text { (upstream) }\end{array} & \begin{array}{l}\text { regulation of } \\ \text { ubiquitin protein } \\ \text { ligase activity } \\ \text { involved in mitotic } \\ \text { cell cycle }\end{array}\end{array}$

CCNA2 (upstream), MAPT (upstream)

FOXM1 (upstream), MAPT (upstream)

protein kinase binding

MAPT (upstream), NUSAP1
(downstream)

MAPT (upstream), NUSAP1 (feedback)

MAPT (upstream), TOP2A

MAPT (upstream), TOP2A (downstream) (upstream)

CDKN3 (feedback), RRM2 (downstream)

CDKN3 (feedback), RRM2 (downstream)

microtubule binding

RRM2 (downstream), HIST1H3H (downstream)

RRM2 (downstream), HIST1H3H (downstream)

enzyme binding

HIST1H3H (downstream), TOP2A (downstream)

TOP2A (upstream), HIST1H3H (downstream)

G1/S transition of mitotic cell cycle

HIST1H3H (downstream), TOP2A
(downstream)

BUB1B (upstream)

TOP2A (upstream), HIST1H3H (downstream)

protein heterotetramerization

\begin{tabular}{ll} 
(downstream) & $\begin{array}{l}\text { (downstream) } \\
\text { BUB1B (upstream) }\end{array}$ \\
\hline
\end{tabular}

protein heterodimerization activity

cellular protein metabolic process

BUB1 mitotic checkpoint serine/threonine kinase B (BUB1B)

MAPT (upstream)

MAPT (upstream)

microtubule associated protein tau (MAPT)

CDKN3 (feedback)

CDKN3 (feedback)

cyclin-dependent kinase inhibitor 3 (CDKN3)

RRM2 (downstream)

RRM2 (downstream)

ribonucleotide reductase regulatory subunit M2 (RRM2)

HIST1H3H (downstream) HIST1H3H (downstream) histone cluster $1 \mathrm{H} 3 \mathrm{~h}$ (HIST1H3H)

NCAPH and RFC4 common tissue distributions were identified Leukemiapromyelocytic(hl60), leukemialymphoblastic(molt4), lymphomaburkittsRaji, Heart, lymphnode, Thyroid, Testis Germ Cell, TestisLeydig Cell from high UNG-activated upstream DAVID GNF_U133A and UNIGENE_EST database. NCAPH and RFC4 different tissue distributions demonstrate Cerebellum, Trachea, BM CD34+, fetallung, salivarygland, PB CD14 + Monocytes, germ cell tumor_disease, PLACENTA, non glioma_disease, CD8 + T 
cells, bronchialepithelialcells, Cardiac Myocytes, infant ( $<3$ years old)_development, PB CD56 + NKCells, Testis Interstitial, embryonic tissue_normal, Thalamus, adrenal tumor_disease, etc. as shown in Table 2. 
Table 2

NCAPH and RFC4 common and different tissue distributions in HCC (HBV or HCV) from our established significant high expression UNG-activated upstream DAVID GNF_U133A_QUARTILE and UNIGENE_EST_QUARTILE database.

\begin{tabular}{|c|c|c|}
\hline \multicolumn{3}{|c|}{ NCAPH and RFC4 common and different tissue distributions in HCC (HBV or HCV) } \\
\hline Common NCAPH and RFC4 & Different NCAPH & Different RFC4 \\
\hline ADIPOCYTE_3rd & Cerebellum_3rd & Trachea_3rd \\
\hline BM CD33 + Myeloid_3rd & BM CD34+_3rd & fetallung_3rd \\
\hline BM CD71 + EarlyErythroid_3rd & salivarygland_3rd & $\begin{array}{l}\text { PB CD14 + } \\
\text { Monocytes_3rd }\end{array}$ \\
\hline lymphomaburkittsRaji_3rd & germ cell tumor_disease_3rd & PLACENTA_3rd \\
\hline Heart_3rd & non glioma_disease_3rd & CD8 + T cells_3rd \\
\hline leukemiapromyelocytic(hl60)_3rd & bronchialepithelialcells_3rd & Cardiac Myocytes_3rd \\
\hline lymphnode_3rd & $\begin{array}{l}\text { infant ( }<3 \text { years } \\
\text { old)_development_3rd }\end{array}$ & PB CD56 + NKCells_3rd \\
\hline CD4 + T cells_3rd & Testis Interstitial_3rd & \\
\hline leukemialymphoblastic(molt4)_3rd & embryonic tissue_normal_3rd & \\
\hline Thyroid_3rd & Thalamus_3rd & \\
\hline Testis Germ Cell_3rd & adrenal tumor_disease_3rd & \\
\hline \multirow[t]{12}{*}{ TestisLeydig Cell_3rd } & $\begin{array}{l}\text { juvenile (< } 17 \text { years } \\
\text { old)_development_3rd }\end{array}$ & \\
\hline & Testis Seminiferous Tubule_3rd & \\
\hline & lymph node_normal_3rd & \\
\hline & testis_normal_3rd & \\
\hline & thymus_normal_3rd & \\
\hline & esophageal tumor_disease_3rd & \\
\hline & esophagus_normal_3rd & \\
\hline & $\begin{array}{l}\text { neonate (< } 4 \text { weeks } \\
\text { old)_development_3rd }\end{array}$ & \\
\hline & bone_normal_3rd & \\
\hline & oral tumor_disease_3rd & \\
\hline & small intestine_normal_3rd & \\
\hline & laryngeal cancer_disease_3rd & \\
\hline
\end{tabular}




\section{Discussion}

NCAPH and RFC4 common molecular subnetworks and the related family members have been reported relationship with cancer including BUB1B (upstream), MAPT (upstream), CDKN3 (feedback), RRM2 (downstream), HIST1 H3H (downstream) in the references. For instance, prevalence of germline mutations in BUB1B in individuals with early-onset colorectal cancer [13]. MAPT is a promising independent prognostic marker and tumor suppressive protein of clear cell renal cell carcinoma [14]. LncRNA TTN-AS1 regulates RRM2 to promote breast cancer progression [15]. KMT2A histone methyltransferase contributes to colorectal cancer development via promoting cathepsin Z transcriptional activation [16].

Our results show the common molecules MAPT interaction with CDKN3 with RRM2 with HIST1H3H; The common biological process and molecular function of MAPT as microtubule binding; $\mathrm{HIST} 1 \mathrm{H} 3 \mathrm{H}$ as cellular protein metabolic process in HCC (HBV or HCV) from our established high UNG-activated upstream GRNInfer, DAVID GOTERM_BP_DIRECT and GOTERM_MF_DIRECT database (Fig. 1-2, Table 1, Supp 4-6). Therefore, we put forward and mutual positively verify protein metabolism in UNG-activated NCAPH and RFC4 subnetworks for viral HCC development via microtubule binding.

Low UNG-activated upstream molecular network in no-tumor hepatitis/cirrhotic tissues (HBV or HCV infection) was identified as NKX2_5 activation to UNG from our established significant low expression UNG activation GRNInfer database (Supp 1-2). Low UNG-activated upstream common biological process and molecular function network shows UNG, CIA01, NKX2_5, REG3A as protein binding; UNG, NKX2_5 as negative regulation of apoptotic process from our established low UNG activation DAVID GOTERM_BP_DIRECT and GOTERM_MF_DIRECT database (Supp 3). We put forward negative regulation of apoptotic process in low UNG-activated upstream network for no-tumor hepatitis/cirrhotic tissues (HBV or HCV infection) development via protein binding.

Our results show the common cellular component of UNG, NCAPH, CDKN3, RRM2, HIST1H3H at nucleus; $\mathrm{NCAPH}, \mathrm{HIST} 1 \mathrm{H} 3 \mathrm{H}$ at membrane in $\mathrm{HCC}(\mathrm{HBV}$ or HCV); The common tissue distributions of NCAPH and RFC4 in Leukemiapromyelocytic(hl60), leukemialymphoblastic(molt4), etc. from our established high UNG-activated upstream DAVID GOTERM_CC_DIRECT, GNF_U133A and UNIGENE_EST database. NCAPH and RFC4 different tissue distributions demonstrate Cerebellum, Trachea, BM CD34+, fetallung, salivarygland, PB CD14 + Monocytes, germ cell tumor_disease, etc. (Fig. 1-2, Table 1-2, Supp 4-6). Therefore, we propose and mutual positively verify membrane protein metabolism in UNG-activated NCAPH and RFC4 subnetworks for viral HCC|blood cancer development via nucleus microtubule binding. The different tissue distributions of NCAPH and RFC4 negatively verify our hypothesis. 
Low UNG-activated upstream common cellular component network demonstrates UNG, NKX2_5 at nucleus from our established low UNG activation DAVID GOTERM_CC_DIRECT database. Low UNGactivated upstream most common tissue distribution network was identified Tonsil from our established low UNG activation DAVID GNF_U133A and UNIGENE_EST database (Supp 3). We put forward negative regulation of apoptotic process in low UNG-activated upstream network for no-tumor hepatitis/cirrhotic tissues (HBV or HCV infection) and tonsil development via nucleus protein binding, and negatively verify our hypothesis.

\section{Conclusion}

We put forward and mutual positively verify membrane protein metabolism in UNG-activated NCAPH and RFC4 subnetworks for viral HCC|blood cancer development via inside-out microtubule binding, and also negatively verify our hypothesis in low UNG-activated upstream network of no-tumor hepatitis/cirrhotic tissues (HBV or HCV infection). Other UNG-activated upstream molecular and knowledge subnetworks containing CDKN3 (feedback) will be computed and the hypotheses proposed for the whole systemic molecular and cellular mechanisms of HCC (HBV or HCV) related cancer development in the future.

\section{Declarations}

\section{Ethics approval and consent to participate}

Not applicable.

\section{Consent for publication}

All authors have approved the manuscript for submission.

\section{Availability of data and materials}

We declare the study data GSE10140-10141 public free from NCBI.

\section{Competing interests}

The authors report no conflicts of financial and non-financial interests.

\section{Funding}

This work is supported by grants from the National Natural Science Key Fondation of China (61433015), National Natural Science Youth Fondation of China (81501372), National Social Science Major Fondation of China (14ZDB154 \& 15ZDB017), and the Independent scientific research project of Tsinghua University (20161080056).

\section{Authors' contributions}


LW designed the whole experiment and wrote the paper. LW \& MJ \& QC analyzed the data and look up in references. JH \& MJ \& ZJ computed CC and GRNInfer. JH \& QC \& HF prepared figures and tables.

\section{Acknowledgments}

This work is supported by grants from the National Natural Science Key Fondation of China (61433015), National Natural Science Youth Fondation of China (81501372), National Social Science Major Fondation of China (14ZDB154 \& 15ZDB017), and the Independent scientific research project of Tsinghua University (20161080056).

\section{References}

1. Wang J, Che W, Wang W, Su G, Zhen T, Jiang Z. CDKN3 promotes tumor progression and confers cisplatin resistance via RAD51 in esophageal cancer. Cancer management research. 2019;11:325364.

2. Li WH, Zhang L, Wu YH: CDKN3 regulates cisplatin resistance to colorectal cancer through TIPE1. European review for medical and pharmacological sciences 2020, 24(7):3614-3623.

3. Xiang J, Fang L, Luo Y, Yang Z, Liao Y, Cui J, Huang M, Yang Z, Huang Y, Fan X, et al. Levels of human replication factor $\mathrm{C} 4$, a clamp loader, correlate with tumor progression and predict the prognosis for colorectal cancer. J Transl Med. 2014;12:320.

4. Kim JT, Cho HJ, Park SY, Oh BM, Hwang YS, Baek KE, Lee YH, Kim HC, Lee HG. DNA Replication and Sister Chromatid Cohesion 1 (DSCC1) of the Replication Factor Complex CTF18-RFC is Critical for Colon Cancer Cell Growth. J Cancer. 2019;10(24):6142-53.

5. Qiu X, Gao Z, Shao J, Li H. NCAPH is upregulated in endometrial cancer and associated with poor clinicopathologic characteristics. Annals of human genetics 2020.

6. Cui F, Hu J, Xu Z, Tan J, Tang H. Overexpression of NCAPH is upregulated and predicts a poor prognosis in prostate cancer. Oncology letters. 2019;17(6):5768-76.

7. Wang Y, Joshi T, Zhang XS, Xu D, Chen L. Inferring gene regulatory networks from multiple microarray datasets. Bioinformatics. 2006;22(19):2413-20.

8. Huang da W, Sherman BT, Lempicki RA. Systematic and integrative analysis of large gene lists using DAVID bioinformatics resources. Nature protocols. 2009;4(1):44-57.

9. Huang da W, Sherman BT, Lempicki RA. Bioinformatics enrichment tools: paths toward the comprehensive functional analysis of large gene lists. Nucleic acids research. 2009;37(1):1-13.

10. Hoshida Y, Villanueva A, Kobayashi M, Peix J, Chiang DY, Camargo A, Gupta S, Moore J, Wrobel MJ, Lerner $\mathrm{J}$, et al. Gene expression in fixed tissues and outcome in hepatocellular carcinoma. N Engl J Med. 2008;359(19):1995-2004.

11. Villanueva A, Hoshida Y, Battiston C, Tovar V, Sia D, Alsinet C, Cornella H, Liberzon A, Kobayashi M, Kumada $\mathrm{H}$, et al. Combining clinical, pathology, and gene expression data to predict recurrence of hepatocellular carcinoma. Gastroenterology. 2011;140(5):1501-12 e1502. 
12. Tusher VG, Tibshirani R, Chu G. Significance analysis of microarrays applied to the ionizing radiation response. Proc Natl Acad Sci U S A. 2001;98(9):5116-21.

13. Hahn MM, Vreede L, Bemelmans SA, van der Looij E, van Kessel AG, Schackert HK, Ligtenberg MJ, Hoogerbrugge N, Kuiper RP, de Voer RM. Prevalence of germline mutations in the spindle assembly checkpoint gene BUB1B in individuals with early-onset colorectal cancer. Genes Chromosomes Cancer. 2016;55(11):855-63.

14. Han X, Sekino Y, Babasaki T, Goto K, Inoue S, Hayashi T, Teishima J, Sakamoto N, Sentani K, Oue N, et al. Microtubule-associated protein tau (MAPT) is a promising independent prognostic marker and tumor suppressive protein in clear cell renal cell carcinoma. Urol Oncol. 2020;38(6):605. e609-605 e617.

15. Feng $H$, Wang $Q$, Xiao W, Zhang B, Jin Y, Lu H. LncRNA TTN-AS1 Regulates miR-524-5p and RRM2 to Promote Breast Cancer Progression. Onco Targets Ther. 2020;13:4799-811.

16. Fang Y, Zhang D, Hu T, Zhao H, Zhao X, Lou Z, He Y, Qin W, Xia J, Zhang X, et al. KMT2A histone methyltransferase contributes to colorectal cancer development by promoting cathepsin $Z$ transcriptional activation. Cancer medicine. 2019;8(7):3544-52.

\section{Figures}




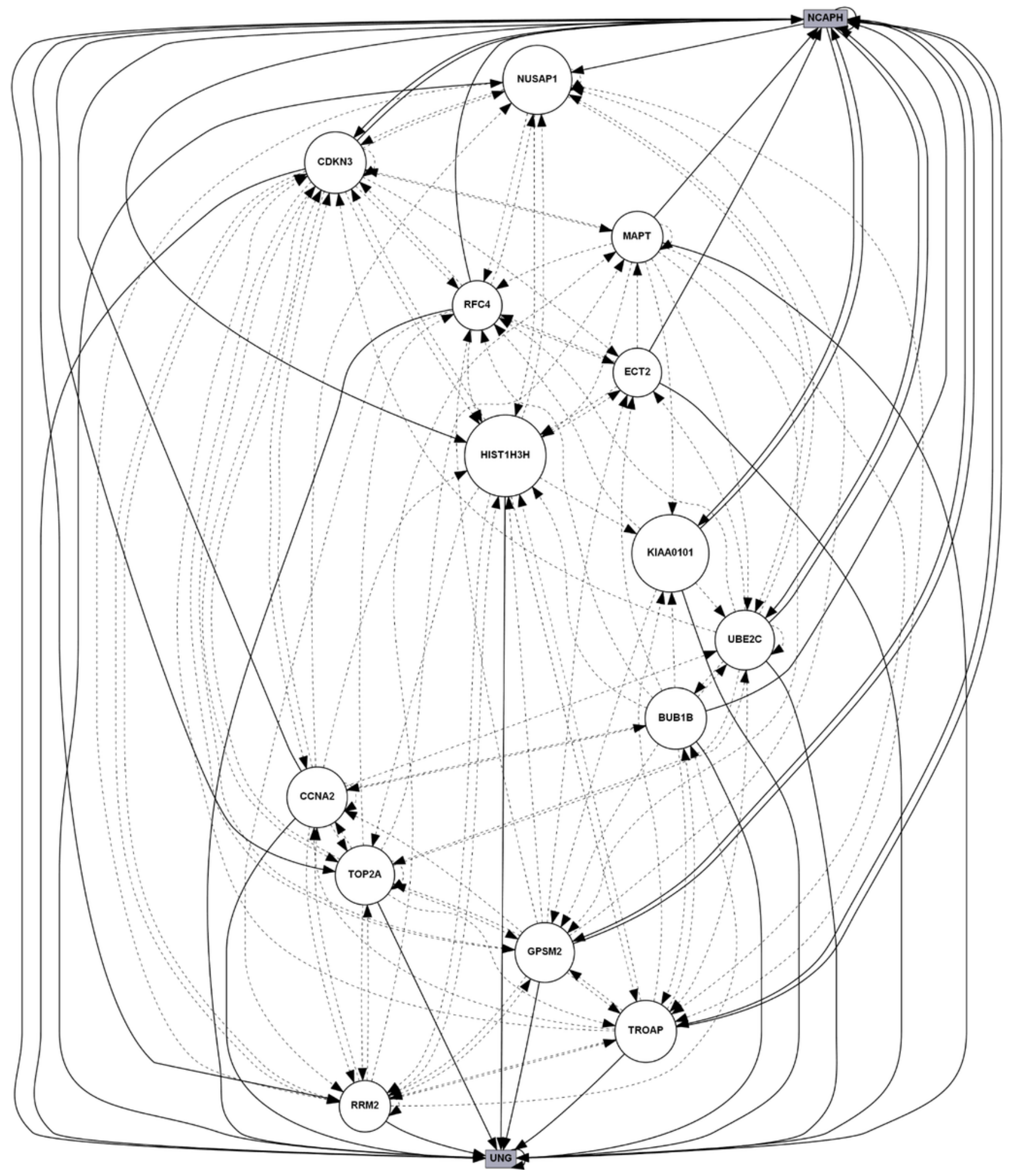

Figure 1

NCAPH direct and indirect molecular subnetwork construction containing CDKN3 (feedback) in HCC (HBV or HCV) from our established significant high expression UNG-activated upstream GRNInfer database. Solid line with black arrow represents direct activation relationships with NCAPH and UNG, respectively. Dashed line with black arrow represents indirect activation relationships with UNG. NCAPH direct and indirect molecular subnetwork construction containing CDKN3 (feedback) in HCC (HBV or HCV) 


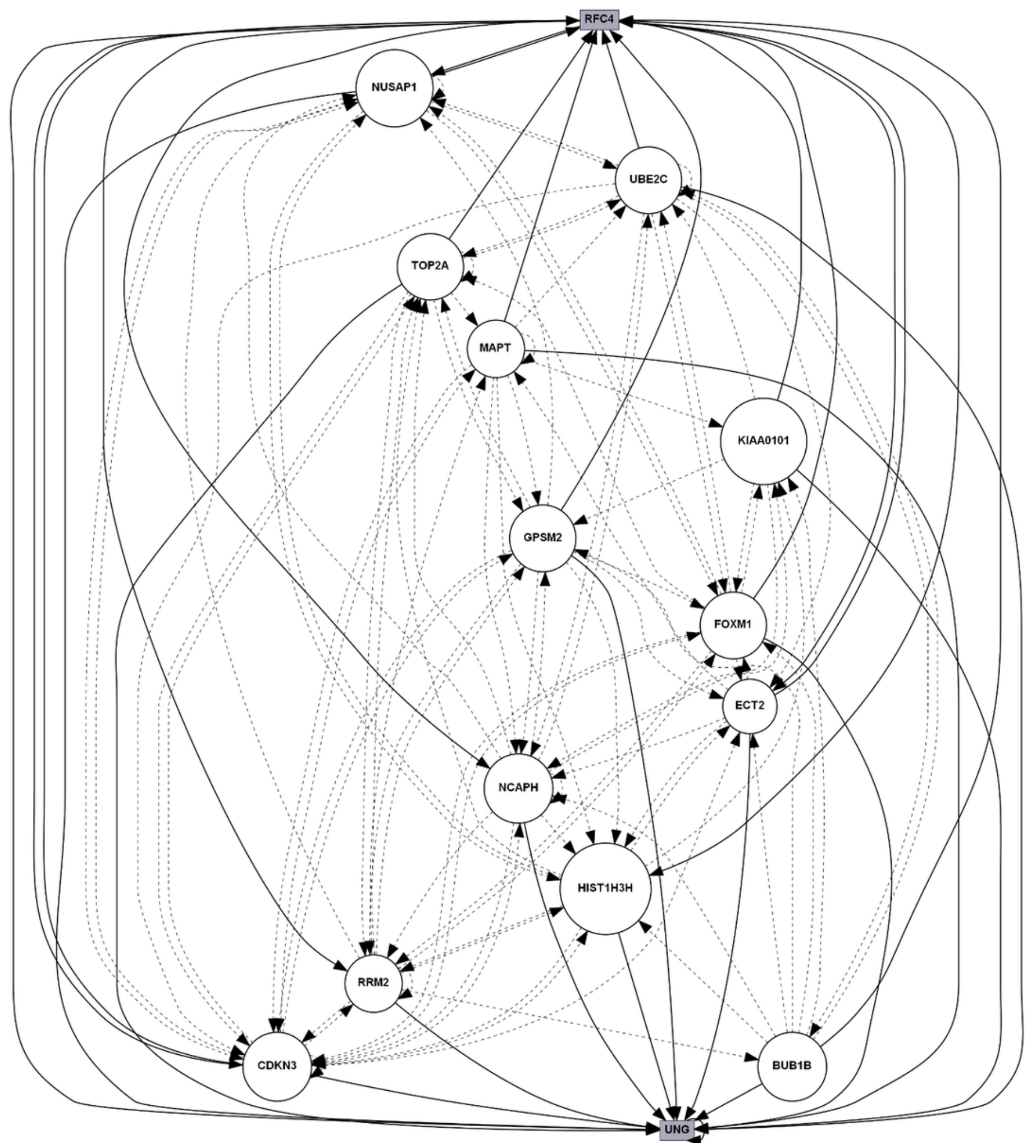

Figure 2

RFC4 direct and indirect molecular subnetwork construction containing CDKN3 (feedback) in HCC (HBV or HCV) from our established significant high expression UNG-activated upstream GRNInfer database. Solid line with black arrow represents direct activation relationships with RFC4 and UNG, respectively. Dashed line with black arrow represents indirect activation relationships with UNG. RFC4 direct and indirect molecular subnetwork construction containing CDKN3 (feedback) in HCC (HBV or HCV) 


\section{Supplementary Files}

This is a list of supplementary files associated with this preprint. Click to download.

- Supplementarymaterial.docx 